\title{
Increased Focal Interictal Discharges During Specific Cognitive Tasks
}

\author{
Olaf Blanke, Alan Pegna, Jean-Pierre Marcoz'1, Theodor Landis and Margitta Seeck \\ Department of Neurology, University Hospital of Geneva, 1211 Geneva, Switzerland and ${ }^{1}$ Department of Paediatrics, \\ Cantonal Hospital, Sion, Switzerland
}

\begin{abstract}
We present a patient with pharmacoresistant right temporal lobe epilepsy who showed a significant increase in focal interictal spikes in response to specific visuospatial tasks. Temporal spiking was particularly increased during immediate recall of visuospatial material, but not during verbal memory tasks. This reflexive phenomenon, situated in between cognitive alterations as seen in most patients with epilepsy and frank seizures elicited by higher cerebral function as in reflex epilepsy, is discussed in relation to cognition and underlying neural substrates.
\end{abstract}

\section{Introduction}

Various non-specific extrinsic techniques to increase interictal spiking or to facilitate seizures, such as hyperventilation, intermittent photic stimulation, and sleep deprivation are well known. In comparison, the increase in interictal or ictal epileptiform discharges by specific cognitive activity is extremely rare (Altafullah and Halgren, 1988; Helmstaedter et al., 1992; Binnie, 1994; Brockway et al., 1996).

Interictal spikes and prolonged rhythmic ictal discharges are both intimately related to the genesis of clinically evident epileptic seizures (Hughes, 1989) and occur infrequently in non-epileptic individuals (Zivin and AjmoneMarsan, 1968). Rarely do highly specific intrinsic stimuli such as complex cognitive tasks (e.g. reading: Bickford et al., 1956; Wolf, 1994) induce seizures which are then referred to as reflex epilepsy. The international classification of seizures and epilepsies (Commission, 1989) considers reflex epilepsy to be a primary localization-related epilepsy.

In contrast to primary epilepsies, clinical or EEG changes in secondary epileptic disorders are associated with the activation of a system of neurons limited to one part of the brain. In patients with partial epilepsies, the cerebral location of the cognitive deficit and of the epileptic focus were shown to converge (Jones-Gotman et al., 1993). It could further be demonstrated that the presence of interictal epileptic discharges during neuropsychological testing can impair the patient's performance (the so-called TCI, transient cognitive impairment: Aarts et al., 1984; Regard et al., 1985, 1994). It is therefore advisable to monitor the EEG during neuropsychological testing in order to deduce meaningful interpretations. Cognition is known to diminish interictal spiking without a specific relation to the cognitive task (Hutt, 1972; Aarts et al., 1984). On the other hand, increase of interictal epileptic discharges during cognitive activities has rarely been reported (Altafullah and Halgren, 1988; Helmstaedter et al., 1992; Binnie, 1994; Brockway et al., 1996).

Here, we present a patient with pharmacoresistant right temporal lobe epilepsy who showed a significant increase of right temporal focal spikes during visuospatial tasks, as compared to verbal material and to various control periods without neuropsychological testing. In this patient, a consistent relationship existed between the occurrence of a limited number of closely related mental activities and the presence of focal discharges.

\section{Materials and methods \\ Case history}

This 21-year-old, right-handed, male biology student began having seizures at the age of 12 years. The habitual seizures started with an aura described as an ascending epigastric sensation, followed by manual and facial automatisms, which lasted 2-4 min. Seizure frequency was 10-20 per month, often followed by secondary generalization. Medical history revealed febrile seizures between the

Correspondence to: Dr M. Seeck, Department of Neurology, Laboratory of Presurgical Evaluation for Epilepsy, University Hospital of Geneva, 24 rue Micheli-du-Crest, 1211 Geneva, Switzerland. Tel: + 4122 3728355, Fax: + 4122 3728340, e-mail: mase@diogenes.hcuge.ch 
ages of 6 months and 4 years. Mono- and polytherapy with carbamazepine, phenytoin, gabapentine, and vigabatrin did not modify his condition. Family history regarding epilepsy was negative. On admission, neurological examination was normal. Presurgical evaluation, including continuous video-EEG recording, MRI, nuclear imaging (PET and SPECT), and neuropsychological examination was performed. Seizure onset, interictal spikes, and continuous slowing were seen over the right temporal region. Postictally, the patient presented a discrete weakness of the left arm and complete amnesia for the event. MRI showed a right hippocampal sclerosis and a dysplasia in the parahippocampal gyrus (Fig. 1a, b). Nuclear imaging (PET and SPECT) confirmed a right temporal dysfunction. The interictal neuropsychological examination was completely normal and in the superior range of an age-matched sample of normal male subjects. The patient underwent a right partial temporolobectomy and has now been seizure free for 18 months. Histopathological examination of the resected tissue confirmed the MRI findings.

\section{EEG data analysis}

EEG was recorded with 31 electrodes, placed according to the 10-20 system, with special coverage of the temporal lobes and bilateral sphenoidal wire electrodes. EEG recordings were continuous, synchronized with the video recordings (bandpass $0.1-70 \mathrm{~Hz}$, sampling rate $128 \mathrm{~Hz}$, fronto-central reference: Deltamed, France). The EEG was visually analysed and sharp waves and spike waves were found over the right temporal region (Sp2, T4, F8), as well as continuous slowing over this area. No epileptogenic abnormality was noted from any other region. Only spikes, spike waves or sharp-slow waves, which were identified in at least one referential and one bipolar montage, were included in the analysis. The synchronized video and EEG recording permitted calculation of the exact duration of each neuropsychological subtest. The spike frequency (spikes per minute) was then determined for each neuropsychological test. Increase of spike frequency was considered to be significant if the calculated value was higher than two standard deviations of the mean of all baseline periods (10 periods of a comparable vigilance level, total length: $26 \mathrm{~min})$. Statistical analysis was performed using the Wilcoxon test for dependent samples.

\section{Neuropsychological examination}

The total duration of interictal testing was $2 \mathrm{~h}$ and $15 \mathrm{~min}$. All applied neuropsychological tests are listed in Table 1. The spike frequency was analysed for the entire period of the neuropsychological examination and during various control periods when a comparable degree of alertness could be assumed (watching TV, conversation, periods between and immediately after neuropsychological tasks). The patient's habitual medication consisted of vigabatrin
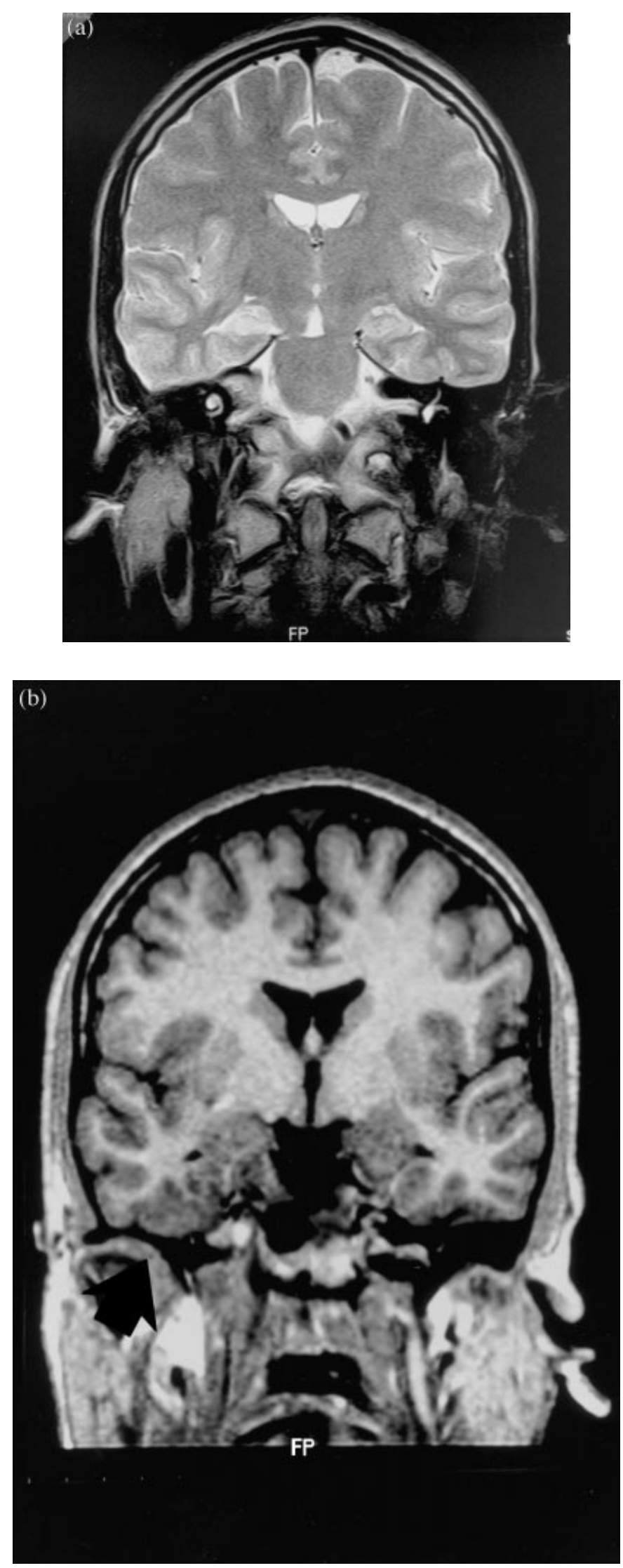

Fig. 1. T2-weighted coronal magnetic resonance scan showing right hippocampal sclerosis (a) and dysplasia in the right parahippocampal gyrus (b). 


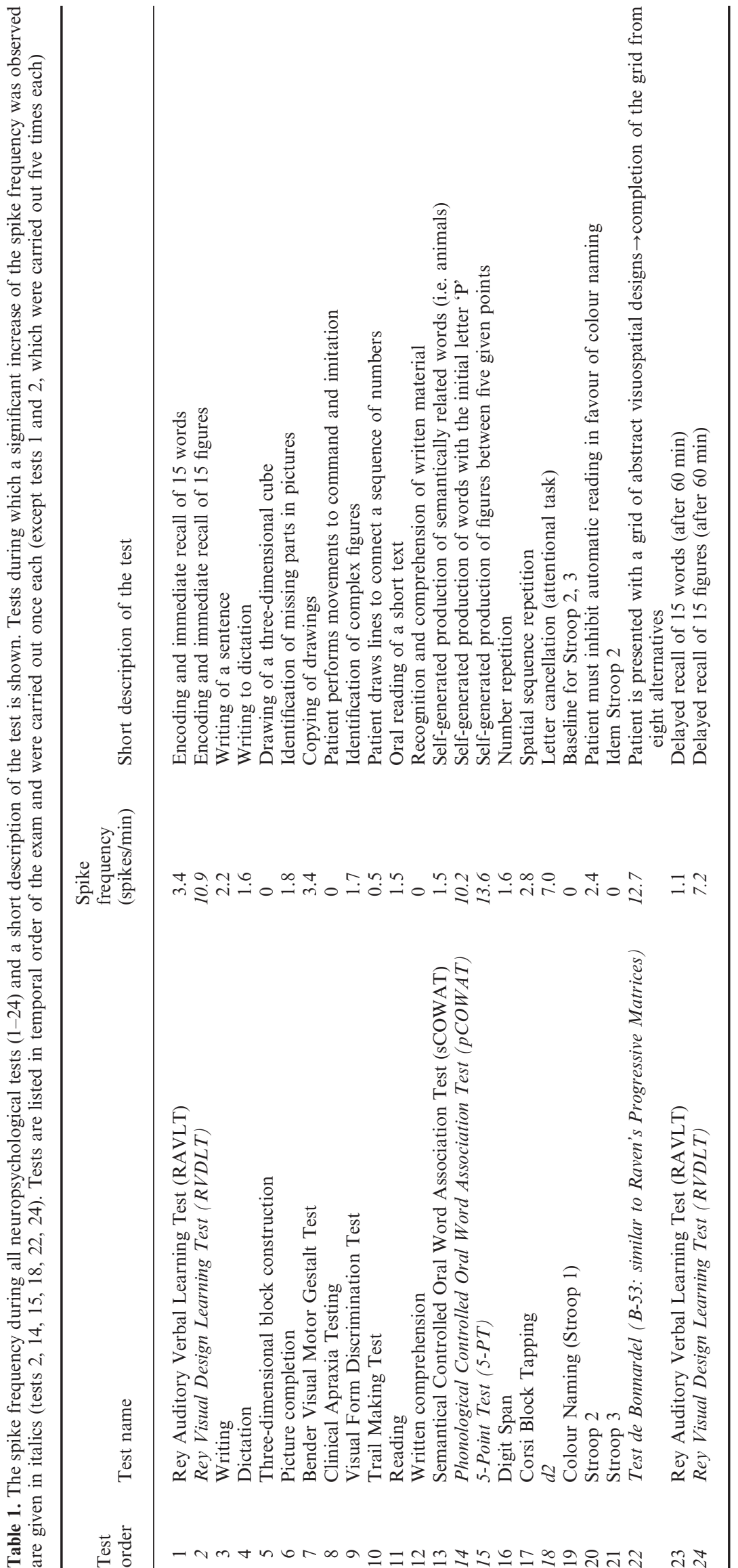




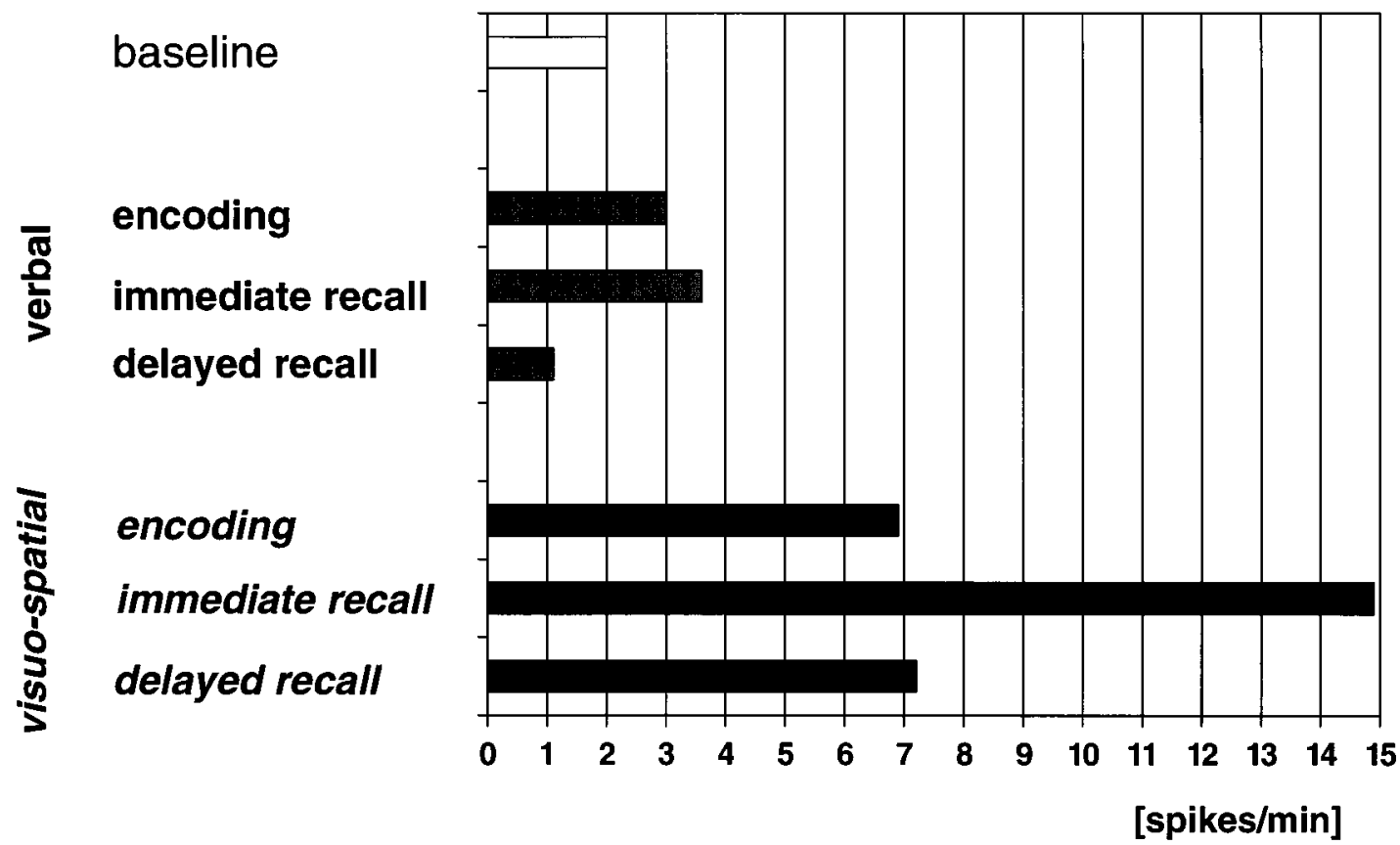

Fig. 2. Mean spike frequency during encoding, immediate and delayed recall of verbal and visuospatial material as compared to the baseline spike frequency.

$(1000 \mathrm{mg} /$ day $)$, gabapentine $(1200 \mathrm{mg} /$ day $)$, and carbamazepine $(2100 \mathrm{mg} /$ day). During hospitalization, only vigabatrin was diminished over the first 2 days to $500 \mathrm{mg} /$ day. During the following 5 days, prior to the neuropsychological examination and baseline periods, no further changes in his medication were carried out.

\section{Results}

No material-specific neuropsychological deficits were found. The overall performance scores of the patient for each test were normal, and in the superior range of age-matched control subjects.

The spike frequency during the various control periods (baseline frequency) was at 2.0/min (SD 1.4; Table 1). However, during five neuropsychological tests, a significant increase of the spike frequency in relation to the baseline spike frequency was noted. Highest spike frequencies were obtained during three visuospatial tasks: immediate recall during the Rey Visual Design Learning Test (RVDLT: 10.9; Rey, 1964, Fig. 2), figural fluency with the 5-point test (5-PT: 13.6; Regard et al., 1982; Table 1), and during the completion of abstract visual designs, the B-53 (B-53: 12.7; Bonnardel, 1977, Table 1).

Presentation of visuospatial material during the RVDLT and of verbal material during the Rey Auditory-Verbal Learning Test (RAVLT) were each carried out five times (encoding), as well as immediate recall. When the encoding and immediate recall period were taken together for verbal and visuospatial material and compared statistically, a highly significant difference in spike frequency $(P=0.01$, $t=1, z=2.7$; mean verbal: 3.4 , SD 3.0, mean visuo- spatial: 10.9, SD 3.7) was found (Fig. 2). During all periods of visuospatial encoding, the average spike frequency showed a significant increase to 6.9 (SD 2.1; $P=0.04$; $z=2.02$ ), during immediate recall of visuospatial material to 14.9 (SD 7.2; $P=0.04 ; z=2.02$ ) (Fig. 2). In contrast, during verbal encoding (mean: 3.0, SD 3.5) and immediate verbal recall (mean: 3.6, SD 3.5), the increase of spike frequency was not significant (encoding: $P=0.89$; recall: $P=0.69)$.

Delayed recall of verbal and visuospatial material were each carried out once, showing a spike frequency of 1.1 (verbal) and 7.2 (visuospatial). When compared with the baseline values, only the spike frequency during delayed visuospatial recall was outside the $2 \mathrm{SD}$ range.

The B-53 was carried out once and consisted of 60 visuospatial analogy problems. During this test, the patient is confronted with a grid of abstract visuospatial designs in which the last one is missing. The patient must then choose among eight alternatives offered as a logical continuation of the grid pattern. During the matching of these visual patterns, the spike frequency rose to 12.7 spikes $/ \mathrm{min}$ (Table 1). An equally important increase of interictal spiking was seen during the self-generated production of figures between five equally spaced points (5-PT; Regard et al., 1982). This test elicited a spike frequency of 13.6 spikes/min (Table 1). The self-generated production of words was carried out once for phonological (phonological Controlled Oral Word Association Test: pCOWAT) and semantical material (semantical Controlled Oral Word Association Test: sCOWAT) and for 2 min each. During the sCOWAT the spike frequency was at $1.5 \mathrm{spikes} / \mathrm{min}$, but rose to 10.1 during its phonological homologue 
(pCOWAT), i.e. outside the $2 \mathrm{SD}$ range of the baseline. During the testing for concentrated attention (d2) the spike frequency was measured at 7.0 spikes/min, which was also beyond the 2 SD level but of moderate increase.

Nine months after his operation, a neuropsychological examination under video-EEG control showed an even better neuropsychological performance and no interictal epileptiform discharges. Since the operation, the patient has reported no seizures.

\section{Discussion}

In this young, right-handed patient with right temporal lobe epilepsy, a major increase of spikes over the right temporal lobe was observed repeatedly during processing of visuospatial material. The fact that all epileptogenic activity disappeared after anterior temporal lobectomy allowed us to conclude that the epileptic generator which became particularly active during visuospatial processing was located within the resected structures. Frequent interictal discharges are known to be associated with dysplasias, as was found in the right parahippocampal gyrus of our patient. The electrophysiological dissociation between the mnestic processing of verbal and visuospatial material clearly demonstrated that cognitive activation of this localized neural circuit during visuospatial encoding and recall led to electrical activation of a neuronal network which included the right temporal epileptic focus, or probably more specifically the parahippocampal gyrus.

Concerning the testing of memory functions, our analysis revealed a significant increase of the spike frequency by visuospatial encoding, as well as by immediate and delayed recall of visuospatial material. This significant increase was observed in comparison to the baseline frequency and verbal memory exams. Among the three visuospatial memory tests, immediate recall elicited the highest values, followed by delayed recall and encoding. It has been shown in numerous studies that the right hemisphere has a prominent role in the analysis of spatial stimulus characteristics (e.g. De Renzi, 1997). Studies of patients with focal brain lesions have shown that the right non-dominant temporal lobe plays an important role in encoding, recognition, and recall of visually presented geometric or Abstract patterns and unfamiliar faces (Warrington, 1982) and that the mesial structures of the right temporal lobe are crucial for the acquisition of new visuospatial material into memory. Inferences from a brain-lesioned patient to normal subjects have to be made with caution, but multiple non-invasive tests of our patient confirmed the usual hemispheric organization concerning lateralization of verbal and non-verbal cognitive functions: our patient was right-handed, had a right-ear advantage in the dichotic listening test, and did not display postictal aphasia. Consistent with a recent study of healthy volunteers by Gabrieli et al. (1997), the right mesial temporal structures in our patient seem to be more intensively involved in immediate recall of visuo- spatial figures than previously thought (e.g. Ungerleider, 1995). Visuospatial encoding and delayed recall seemed to be based on a more extensive network of which the right mesial temporal lobe was just one component.

When testing executive functions, our results show a significant increase of the spike frequency during the spontaneous production of visuospatial material (5-PT). Regard et al. (1982) showed that visuospatial fluency depends upon the functioning of the right frontal lobe. Patients with right frontal lesions perform more poorly than left frontally brain-damaged patients in the production of figures. Our results suggest that the right temporal lobe is also significantly implicated in this task.

There is still controversy with regard to the cortical structures implicated in semantically and phonologically related word production. Studies with patients having suffered from frontal brain damage showed that phonological word production is deficient after left frontal lesions, whereas the semantical word production is impaired subsequent to any brain lesion in the dominant hemisphere (Jones-Gotman et al., 1993). During similar phonological verbal fluency tasks as used here, these findings were only partly reproduced in nuclear imaging studies (Frith et al., 1991; Boivin et al., 1992; Cardebat et al., 1996) and widespread activation and inhibition patterns were found. In our case, semantically related words did not increase right temporal spiking, whereas phonological word processing did. Our results provide further evidence that the spontaneous production of words within semantical or phonological categories activates different neuronal circuits. They further suggest that the right temporal lobe, including the right parahippocampus, can be more intensively involved in phonological word generation than previously believed. This effect may be due to alternative word generation strategies (e.g. covert visual imagery) used by the patient during the phonological fluency task.

The fact that cognitive activity during processing of visuospatial analogy problems (B-53), presumably a right hemispheric test (i.e. Villardita, 1985), did increase right temporal spiking suggests that visuospatial reasoning also relies on right temporal structures.

During most visuospatial tests, a significant increase of the spike frequency occurred in our patient. This specificity suggests a neurophysiological mechanism similar to those underlying reflex epilepsy (i.e. Wolf, 1994). In reflex epilepsy, one specific cognitive task activates a specific neural network which then elicits generalized or focal seizures in a repetitive manner. In our patient, we showed a well lateralized and localized epileptogenic EEG-pattern without obvious seizures or cognitive impairment in a materialspecific mode. This phenomenon is situated in between an unchanged EEG during specific cognitive tasks as is seen in most patients with partial and generalized epilepsy, and frank seizures elicited by specific cognitive activities in patients suffering from primary localization-related reflex epilepsy. 
This case also underlines the potential of discrete brain regions (here, the right temporal lobe) to participate actively in an interconnected cerebral network in which many components, local and remote, are activated depending upon the type of cognitive processing (Mesulam, 1990). Continuous video-EEG monitoring during neuropsychological examinations of epileptic patients permits the identification of the few patients who reveal specific relationships between a well-defined focal EEG pattern and cognitive tasks. Quantification of this electrocognitive relationship may help to describe the principal relays of distributed neuronal assemblies implicated in a specific cognitive activity.

\section{Acknowledgements}

This work was supported by the Swiss National Science Foundation (Grant No. 31-52933.97).

\section{References}

Aarts JHP, Binnie CD, Smit AM, Wilkins AJ. Selective cognitive impairment during focal and generalized epileptiform EEG activity. Brain 1984; 107: 293-308.

Altafullah I, Halgren E. Focal medial temporal lobe spike-wave complexes evoked by a memory task. Epilepsia 1988; 29: 8-13.

Bickford RG, Whalen JL, Klaas DW. Reading epilepsy. Trans American Neurological Association 1956; 81: 100-2.

Binnie CD. Cognitive impairment - is it inevitable? Seizure 1994; 3: 17-22.

Boivin MJ, Giordani B, Berent S, Amato DA, Lehtinen S, Koeppe, RA, Buchtel, HA, Foster NL, Kuhl DE. Verbal fluency and positron emission tomographic mapping of regional cerebral glucose metabolism. Cortex 1992; 28: 231-9.

Bonnardel R. Test d'intelligence non-verbal-b-53-Manuel. Issy-lesMoulineaux: Editions Scientifiques et Psychotechniques, 1977.

Brockway JP, Follmer RL, Burrows GS, Solsrud KA, Preuss LA. EEG spindle bursts from hippocampus. Neurocase 1996; 2: 251-8.

Cardebat D, Démonet JF, Viallard G, Faure S, Puel M, Celsis P. Brain functional profiles in formal and semantic fluency tasks: a SPECT study in normals. Brain and Language 1996; 52: 305-13.
Commission on Classification and Terminology of the ILAE. Proposal for revised classification of epilepsies and epileptic syndromes. Epilepsia 1989; 30: 389-99.

De Renzi E. Visuospatial and constructional disorders. In: Feinberg TE, Farah MJ, editors. Behavioural neurology and neuropsychology. New York: McGraw Hill, 1997: 297-308.

Frith CD, Friston KJ, Liddle PF, Frackowiak RSJ. A PET study of word finding. Neuropsychologia 1991; 29: 1137-48.

Gabrieli JDE, Brewer JB, Desmond JE, Glover GH. Separate neural bases of two fundamental memory processes in the human medial temporal lobe. Science 1997; 276: 264-6.

Helmstaedter C, Hufnagel A, Elger CE. Seizures during cognitive testing in patients with temporal lobe epilepsy: possibility of seizure induction by cognitive activation. Epilepsia 1992; 33: 892-7.

Hughes JR. The significance of the interictal spike discharge: a review. Journal of Clinical Neurophysiology 1989; 6: 207-26.

Hutt SJ. Experimental analysis of brain activity and behaviour in children with 'minor' seizures. Epilepsia 1972; 13: 520-34.

Jones-Gotman M, Smith ML, Zatorre RJ. Neuropsychological testing for localizing and lateralizing the epileptogenic region. In: Engel J, editor. Surgical treatment of the epilepsies, 2nd edn. New York: Raven Press, 1993: 245-61.

Mesulam MM. Large-scale neurocognitive networks and distributed processing for attention, language, and memory. Annals of Neurology 1990; 28: 597-613.

Regard M, Strauss E, Knapp P. Children's production on verbal and non-verbal fluency tasks. Perception and Motor Skills 1982; 55: 939-44.

Regard M, Landis T, Wieser HG, Hailemariam S. Functional inhibition and release: unilateral tachistoscopic performance and stereoencephalographic activity in a case with left limbic status epilepticus. Neuropsychologia 1985 ; 23: 575-81.

Regard M, Cook, ND, Wieser HG, Landis, T. The dynamics of cerebral dominance during unilateral limbic seizures. Brain 1994; 117: 91-104.

Rey A. L'examen clinique en psychologie. Paris: Presses Universitaires de France, 1964: 141-9.

Ungerleider LG. Functional brain imaging studies of cortical mechanisms for memory. Science 1995; 270: 769-75.

Villardita C. Raven's progressive matrices and intellectual impairment in patients with focal brain damage. Cortex 1985; 21: 627-34.

Warrington EK. Neuropsychological studies of object recognition. Philosophical Transactions of the Royal Society of London 1982; 298B: 15-93.

Wolf P. Reading epilepsy. In: Wolf $\mathrm{P}$, editor. Epileptic seizures and syndromes. London: John Libbey, 1994: 67-73.

Zivin L, Ajmone-Marsan C. Incidence and prognostic significance of 'epileptiform' activity in the EEG of non-epileptic patients. Brain 1968; 91: 751-78.

Received on 20 April, 1998; resubmitted on 23 April, 1998; accepted on 23 April, 1998 


\title{
Increased focal interictal discharges
} during specific cognitive tasks

\section{O. Blanke, A. Pegna, J.-P. Marcoz, T. Landis and M. Seeck}

\begin{abstract}
We present a patient with pharmacoresistant right temporal lobe epilepsy who showed a significant increase of focal interictal spikes in response to specific visuospatial tasks. Temporal spiking was particularly increased during immediate recall of visuospatial material, but not during verbal memory tasks. This reflexive phenomenon, situated in between cognitive alterations as seen in most patients with epilepsy and frank seizures elicited by higher cerebral function as in reflex epilepsy, is discussed in relation to cognition and underlying neural substrates.
\end{abstract}

\section{Journal}

Neurocase 1999; 5: 13-9

Neurocase Reference Number:

O137

Primary diagnosis of interest

Pharmacoresistant temporal lobe epilepsy

\section{Author's designation of the case}

None

Key theoretical issue

- Significant increase of right temporal interictal spikes in response to specific visuospatial tasks

Key words: visuospatial memory; interictal spike frequency; reflex epilepsy

Scan, EEG and related measures

EEG, MRI, PET, SPECT

\section{Standardized assessment}

Rey Auditory Verbal Learning Test, Rey Visual Design Learning Test, Semantical Controlled Oral Word Association Test, Phonological Controlled Oral Word Association Test, 5-Point Test, Digit Span, d2, Stroop (1-3), Test de Bonnardel

\section{Lesion location}

- Right hippocampus and right parahippocampal gyrus

Lesion type

Hippocampal sclerosis and parahippocampal dysplasia

\section{Language}

English 